\title{
EMBEDDING ESTIMATES AND FRACTIONAL SMOOTHNESS
}

\author{
WILLIAM BECKNER \\ "we shall begin by studying the fractional powers of the Laplacian" — Eli Stein
}

\begin{abstract}
A short intrinsic proof is given for the Bourgain-Brezis-Mironescu theorem with an extension for higher-order gradient forms. This argument illustrates the role of functional geometry and Fourier analysis for obtaining embedding estimates. New Hausdorff-Young inequalities are obtained for fractional embedding as an extension of the classical Aronszajn-Smith formula. These results include bilinear fractional embedding as suggested by the Landau collision operator in plasma dynamics.
\end{abstract}

Functional forms that characterize smoothness lie at the heart of understanding and rigorously describing the many-body interactions that determine the behavior of dynamical phenomena. Coupled with the establishment of sharp embedding estimates, our overall objective is to expand the working framework for $n$-dimensional Fourier analysis while gaining new insight into uncertainty, restriction phenomena and the role of geometric symmetry. Efforts to obtain optimal constants bring out new features of exact model problems, encoded geometric information and precise lower-order effects.

\section{Bourgain-Brezis-Mironescu theorem}

Let $\Lambda_{\alpha}=\left(-\Delta / 4 \pi^{2}\right)^{\alpha / 2}, \alpha>0,1 \leq p<n / \beta$ and $0<\beta<1$; the main purpose here is to establish embedding estimates for the Besov norms:

$$
\begin{gathered}
\int_{\mathbb{R}^{n} \times \mathbb{R}^{n}} \frac{|f(x)-f(y)|^{p}}{|x-y|^{n+p \beta}} d x d y \rightsquigarrow \int_{\mathbb{R}^{n} \times \mathbb{R}^{n}} \frac{|(\nabla f)(x)-(\nabla f)(y)|^{p}}{|x-y|^{n+p \beta}} d x d y \\
\rightsquigarrow \int_{\mathbb{R}^{n} \times \mathbb{R}^{n}} \frac{\left|\left(\Lambda_{\alpha} f\right)(x)-\left(\Lambda_{\alpha} f\right)(y)\right|^{p}}{|x-y|^{n+p \beta}} d x d y
\end{gathered}
$$

The intrinsic character of these norms captures the interplay between dilation and translation on $\mathbb{R}^{n}$. Classically such norms measure how differentiability controls function size and determines restriction behavior.

The Bourgain-Brezis-Mironescu theorem corresponds to the estimate for $0<\beta<1$ and $1 \leq p<n / \beta([15],[16],[31$ ] and p. 521 in [30]):

$$
\int_{\mathbb{R}^{n} \times \mathbb{R}^{n}} \frac{|f(x)-f(y)|^{p}}{|x-y|^{n+p \beta}} d x d y \geq c\left(\int_{\mathbb{R}^{n}}|f|^{q} d x\right)^{p / q}, \quad q=\frac{p n}{n-p \beta}
$$

For $p=2$, the best constant $c$ is calculated by the author in [11]:

$$
\frac{n-2 \beta}{\beta(1-\beta)} \pi^{\beta+\frac{n}{2}} \frac{\Gamma(2-\beta)}{\Gamma\left(\frac{n}{2}+1-\beta\right)}\left[\frac{\Gamma\left(\frac{n}{2}\right)}{\Gamma(n)}\right]^{2 \beta / n}
$$

This result extends to include fractional powers of the Laplacian: 
Theorem 1. For $f \in \mathcal{S}\left(\mathbb{R}^{n}\right), 0<\beta<1$ and $1 \leq p<n /(\alpha+\beta)$

$$
\int_{\mathbb{R}^{n} \times \mathbb{R}^{n}} \frac{\left|\left(\Lambda_{\alpha} f\right)(x)-\left(\Lambda_{\alpha} f\right)(y)\right|^{p}}{|x-y|^{n+p \beta}} d x d y \geq c\left(\int_{\mathbb{R}^{n}}|f|^{q^{*}} d x\right)^{p / q^{*}}, \quad q^{*}=\frac{p n}{n-p(\alpha+\beta)}
$$

Theorem 2. For $p=2<n /(\alpha+\beta)$, the value of $c$ in Theorem 1 is given by

$$
c=\frac{2}{\beta(1-\beta)} \pi^{\beta-\alpha+\frac{n}{2}} \frac{\Gamma(2-\beta)}{\Gamma\left(\frac{n}{2}+\beta\right)} \frac{\Gamma\left(\frac{n}{2}+\alpha+\beta\right)}{\Gamma\left(\frac{n}{2}-\alpha-\beta\right)}\left[\frac{\Gamma\left(\frac{n}{2}\right)}{\Gamma(n)}\right]^{2(\alpha+\beta) / n}
$$

Proof of Theorem 1. This result follows by application of four lemmas utilizing symmetrization, Stein-Weiss techniques and the general Hardy-Littlewood-Sobolev inequality. Set $g=\Lambda_{\alpha} f$.

STEP 1: apply the Symmetrization Lemma below to obtain

$$
\int_{\mathbb{R}^{n} \times \mathbb{R}^{n}} \frac{|g(x)-g(y)|^{p}}{|x-y|^{n+p \beta}} d x d y \geq \int_{\mathbb{R}^{n} \times \mathbb{R}^{n}} \frac{\left|g^{*}(x)-g^{*}(y)\right|^{p}}{|x-y|^{n+p \beta}} d x d y
$$

where $g^{*}$ is the equimeasurable radial decreasing rearrangement of $|g|$ on $\mathbb{R}^{n}$.

SteP 2: use Lemma 1 below (see Theorem 4.1 in [11]) to obtain

$$
\int_{\mathbb{R}^{n} \times \mathbb{R}^{n}} \frac{\left|g^{*}(x)-g^{*}(y)\right|^{p}}{|x-y|^{n+p \beta}} d x d y \geq D_{p, \beta} \int_{\mathbb{R}^{n}}|x|^{-p \beta}\left|g^{*}(x)\right|^{p} d x
$$

with

$$
D_{p, \beta}=\left.\left.\int_{\mathbb{R}^{n}}|1-| x\right|^{-\lambda}\right|^{p}|x-\eta|^{-n-p \beta} d x
$$

for $\lambda=(n-p \beta) / p=n / q$ and $\eta \in S^{n-1}$.

STEP 3: recall that $q=p n /(n-p \beta)$ and let $\sigma\left(S^{n}\right)$ denote the surface area of the $n$-dimensional unit sphere. Since $g^{*}(x)$ is non-negative and radial decreasing

$$
g^{*}(x) \leq c|x|^{-n / q}, \quad c=\left[n / \sigma\left(S^{n-1}\right)\right]^{1 / q}\left\|g^{*}\right\|_{L^{q}\left(\mathbb{R}^{n}\right)}
$$

and

$$
\begin{aligned}
\int_{\mathbb{R}^{n}}|x|^{-p \beta}\left|g^{*}(x)\right|^{p} d x & \geq\left[\frac{\sigma\left(S^{n-1}\right)}{n}\right]^{p \beta / n}\left[\int_{\mathbb{R}^{n}}\left|g^{*}(x)\right|^{q} d x\right]^{p / q} \\
& =\left[\frac{\sigma\left(S^{n-1}\right)}{n}\right]^{p \beta / n}\left[\int_{\mathbb{R}^{n}}\left|\Lambda_{\alpha} f\right|^{q} d x\right]^{p / q}
\end{aligned}
$$

STEP 4: now for $0<\alpha<(n-p \beta) / p$

$$
\left[\int_{\mathbb{R}^{n}}\left|\Lambda_{\alpha} f\right|^{q} d x\right]^{p / q} \geq c\left[\int_{\mathbb{R}^{n}}|f|^{q^{*}} d x\right]^{p / q^{*}}
$$

if the Hardy-Littlewood-Sobolev inequality holds for

$$
\left\|\frac{1}{|x|^{n-\alpha}} * f\right\|_{L^{q^{*}\left(\mathbb{R}^{n}\right)}} \leq c\|f\|_{L^{q}\left(\mathbb{R}^{n}\right)}
$$

with $c$ a generic constant and $1 / q^{*}=1 / q-\alpha / n$ which holds for $q=n p /(n-p \beta)$ and $q^{*}=n p /(n-p(\alpha+\beta))$.

This completes the proof of Theorem 1 . 
Remark. One could look for an alternate proof using Lemma 1 but without first applying symmetrization. The idea would be to use a Stein-Weiss argument (see Appendix to [10]) depending on Young's inequality for convolution. But the singularity in the convolution kernel is too strong to effectively use such a simple approach.

Symmetrization Lemma. For $f, g$ measurable functions on $\mathbb{R}^{n}$ with $f^{*}, g^{*}$ denoting the equimeasurable radial decreasing rearrangement of $|f|,|g| ; K(x)$ radial decreasing and $p \geq 1$

$$
\int_{\mathbb{R}^{n} \times \mathbb{R}^{n}} K(x-y)|f(x)-g(y)|^{p} d x d y \geq \int_{\mathbb{R}^{n} \times \mathbb{R}^{n}} K(x-y)\left|f^{*}(x)-g^{*}(y)\right|^{p} d x d y
$$

More generally, for $\rho$ a radial monotone increasing function and $\varphi$ a convex function on $\mathbb{R}$ satisfying

(1) $\varphi(t) \geq 0, \varphi(0)=0$

(2) $\varphi$ convex and monotone increasing, $\varphi^{\prime \prime}(t) \geq 0$

(3) $t \varphi^{\prime}(t)$ convex

$$
\int_{\mathbb{R}^{n} \times \mathbb{R}^{n}} K(x-y) \varphi\left[\frac{|f(x)-g(y)|}{\rho(x-y)}\right] d x d y \geq \int_{\mathbb{R}^{n} \times \mathbb{R}^{n}} K(x-y) \varphi\left[\frac{\left|f^{*}(x)-g^{*}(y)\right|}{\rho(x-y)}\right] d x d y
$$

The development of this lemma was outlined in the author's paper [5] and motivated by earlier ideas of Ahlfors [1] (see Lemma 2.2, pages 34-35) perhaps inspired by Hardy and Littlewood [24] but expanding on themes in Hardy, Littlewood and Pólya [25]; also see Baernstein-Taylor [3]. Details of the proof are given in the Appendix below.

Application of the symmetrization lemma is necessary for Step 3 in the proof of Theorem 1 . But generally there is no difficulty in making a reduction to radial functions for the embedding forms described here.

Reduction Lemma. Let "translation" be a generic representation for any transitive action on a manifold for which the volume form is invariant. Then for $K$ non-negative and integrable and $1 \leq p<\infty$

$$
\int_{M \times M} K(u-v)|f(u)-g(v)|^{p} d u d v \geq \int_{M} K(u) d u\left|\|f\|_{L^{p}(M)}-\|g\|_{L^{p}(M)}\right|^{p}
$$

Proof.

$$
\begin{gathered}
\int_{M \times M} K(u-v)|f(u)-g(v)|^{p} d u d v=\int_{M} K(u)\left[\int_{M}|f(u+v)-g(v)|^{p} d v\right] d u \\
\geq \int_{M} K(u) d u\left|\|f\|_{L^{p}(M)}-\|g\|_{L^{p}(M)}\right|^{p}
\end{gathered}
$$

By using $\xi \cdot \eta=\xi \cdot\left(R_{\eta} \hat{e}\right)=\left(R_{\eta}^{-1} \xi\right) \cdot \hat{e}$ on $S^{n-1}$, one obtains from this reduction lemma

$$
\int_{\mathbb{R}^{n} \times \mathbb{R}^{n}} K(x-y)|f(x)-g(y)|^{p} d x d y \geq \int_{\mathbb{R}^{n} \times \mathbb{R}^{n}} K(x-y)|F(x)-G(y)|^{p} d x d y
$$

where

$$
F(x)=\left[\int_{S^{n-1}}|f(|x| \xi)|^{p} d \xi\right]^{1 / p} \quad, \quad G(y)=\left[\int_{S^{n-1}}|g(|y| \xi)|^{p} d \xi\right]^{1 / p}
$$

with $d \xi$ denoting standard surface measure on the sphere. 
Lemma 1. Let $f \in \mathcal{S}\left(\mathbb{R}^{n}\right), 0<\beta<1$ and $1 \leq p<n / \beta$; then

$$
\begin{gathered}
\int_{\mathbb{R}^{n} \times \mathbb{R}^{n}} \frac{|f(x)-f(y)|^{p}}{|x-y|^{n+p \beta}} d x d y \geq D_{p, \beta} \int_{\mathbb{R}^{n}}|x|^{-p \beta}|f(x)|^{p} d x \\
D_{p, \beta}=\left.\left.\int_{\mathbb{R}^{n}}|1-| x\right|^{-\lambda}\right|^{p}|x-\eta|^{-n-p \beta} d x
\end{gathered}
$$

for $\lambda=(n-p \beta) / p$ and $\eta \in S^{n-1}$.

Proof. The first step is to apply the symmetrization lemma to obtain

$$
\int_{\mathbb{R}^{n} \times \mathbb{R}^{n}} \frac{|f(x)-f(y)|^{p}}{|x-y|^{n+p \beta}} d x d y \geq \int_{\mathbb{R}^{n} \times \mathbb{R}^{n}} \frac{\left|f^{*}(x)-f^{*}(y)\right|^{p}}{|x-y|^{n+p \beta}} d x d y .
$$

Then set $t=|x|, s=|y|, h(t)=|x|^{n / p-\beta} f^{*}(x)$. Then inequality (10) for $f^{*}$ will be equivalent to the inequality on the multiplicative group $\mathbb{R}_{+}$:

$$
\begin{gathered}
\int_{\mathbb{R}_{+} \times \mathbb{R}_{+}}|g(x / t) h(t)-g(t / s) h(s)|^{p} \psi(s / t) \frac{d s}{s} \frac{d t}{t} \\
\geq D_{p, \beta} \int_{\mathbb{R}_{+}}|h(t)|^{p} \frac{d t}{t}
\end{gathered}
$$

where $g(t)=t^{(n-p \beta) / 2 p}$,

$$
\psi(t)=\int_{S^{n-1}}\left[t+\frac{1}{t}-2 \xi_{1}\right]^{-(n+p \beta) / 2} d \xi
$$

and $d \xi$ denotes standard surface measure on $S^{n-1}$. Note that $\psi$ is symmetric under inversion. Apply the "triangle inequality lemma" below using $g \psi^{1 / p}$ as the second function in the lemma, and one finds that

$$
\begin{aligned}
D_{p, \beta} & =\int_{\mathbb{R}_{+}}\left|t^{\lambda / 2}-t^{-\lambda / 2}\right|^{p} \psi(t) \frac{d t}{t} \\
& =\left.\left.\int_{\mathbb{R}^{n}}|1-| x\right|^{-\lambda}\right|^{p}|x-\eta|^{-n-p \beta} d x
\end{aligned}
$$

where $\lambda=(n-p \beta) / p$ and $\eta \in S^{n-1}$. Since the determination of $D_{p, \beta}$ depends only on application of the "triangle inequality", the constant must be optimal as observed by a suitable variation of functions in that inequality. To finish the proof of the Lemma 1, observe the result that

$$
\begin{gathered}
\int_{\mathbb{R}^{n} \times \mathbb{R}^{n}} \frac{|f(x)-f(y)|^{p}}{|x-y|^{n+p \beta}} d x d y \geq \int_{\mathbb{R}^{n} \times \mathbb{R}^{n}} \frac{\left|f^{*}(x)-f^{*}(y)\right|^{p}}{|x-y|^{n+p \beta}} d x d y \\
\geq D_{p, \beta} \int_{\mathbb{R}^{n}}|x|^{-p \beta}\left|f^{*}(x)\right|^{p} d x \geq D_{p, \beta} \int_{\mathbb{R}^{n}}|x|^{-p \beta}|f(x)|^{p} d x
\end{gathered}
$$

Lemma 2 (Triangle Inequality). For $f, g, h \in L^{p}\left(\mathbb{R}^{m}\right), 1 \leq p<\infty$

$$
\begin{aligned}
\int_{\mathbb{R}^{m} \times \mathbb{R}^{m}}|g(y-x) f(x)-h(x-y) f(y)|^{p} d x d y \\
\geq \int_{\mathbb{R}^{m}}|| g(y)|-| h(-y)||^{p} d y \int_{\mathbb{R}^{m}}|f(x)|^{p} d x
\end{aligned}
$$


Proof. By a change of variables in $y$ and using the triangle inequality for norms in the first variable on $\mathbb{R}^{n}$ :

$$
\begin{aligned}
\int_{\mathbb{R}^{m} \times \mathbb{R}^{m}}|g(y-x) f(x)-h(x-y) f(y)|^{p} d x d y \\
\quad=\int_{\mathbb{R}^{m} \times \mathbb{R}^{m}}|g(y) f(x)-h(-y) f(x+y)|^{p} d x d y \\
=\int_{\mathbb{R}^{m}}\left\{\left(\int_{\mathbb{R}^{m}}|g(y) f(x)-h(-y) f(x+y)|^{p} d x\right)^{1 / p}\right\}^{p} d y \\
\geq \int_{\mathbb{R}^{m}}\left\{|| g(y)\left|\|f\|_{L^{p}\left(\mathbb{R}^{m}\right)}-\right| h(-y) \mid\|f\|_{\left.L^{p}\left(\mathbb{R}^{m}\right) \mid\right\}^{p} d y}\right. \\
=\int_{\mathbb{R}^{m}}|| g(y)|-| h(-y)||^{p} d y \int_{\mathbb{R}^{m}}|f(x)|^{p} d x .
\end{aligned}
$$

By considering $g, h \geq 0$ and the family $\varepsilon^{m / p} f(\varepsilon x)$, one observes that the inequality is optimal.

A slightly more general form of Lemma 1 can be given that reflects the general Stein-Weiss structure for fractional integrals (see the appendix in [5]) and uses an analogous proof (though depending on radial reduction rather than symmetrization):

Stein-Weiss Lemma. Suppose $K$ is a non-negative symmetric kernel defined on $\mathbb{R}^{n} \times \mathbb{R}^{n}$, continuous on any domain that excludes the diagonal, homogeneous of degree $-n-\gamma, K(\delta u, \delta v)=$ $\delta^{-n-\gamma} K(u, v), 0<\gamma<\min (n, p)$, and $K(R u, R v)=K(u, v)$ for any $R \in S O(n)$. Then for $f \in \mathcal{S}\left(\mathbb{R}^{n}\right)$ and $p \geq 1$,

$$
\begin{gathered}
\int_{\mathbb{R}^{n} \times \mathbb{R}^{n}}|f(x)-f(y)|^{p} K(x, y) d x d y \geq D_{p, \gamma} \int_{\mathbb{R}^{n}}|x|^{-\gamma}|f(x)|^{p} d x \\
D_{p, \gamma}=\left.\left.\int_{\mathbb{R}^{n}}|1-| x\right|^{-\lambda}\right|^{p} K(x, \eta) d x
\end{gathered}
$$

for $\lambda=(n-\gamma) / p$ and $\eta \in S^{n-1}$. This constant is optimal. But note that there is no assumption made that it is finite.

The last lemma required for the proof of Theorem 1 is the Hardy-Littlewood-Sobolev inequality.

Hardy-Littlewood-Sobolev Inequality. For $f \in L^{p}\left(\mathbb{R}^{n}\right), 0<\alpha<n, 1<p<n / \alpha$ and $1 / q=1 / p-\alpha / n$, then

$$
\left\|\frac{1}{|x|^{n-\alpha}} * f\right\|_{L^{q}\left(\mathbb{R}^{n}\right)} \leq c\|f\|_{L^{p}\left(\mathbb{R}^{n}\right)}
$$

and equivalently

$$
\|f\|_{L^{q}\left(\mathbb{R}^{n}\right)} \leq c\left\|\Lambda_{\alpha} f\right\|_{L^{p}\left(\mathbb{R}^{n}\right)}
$$

where $c$ is a generic constant.

A standard proof of this inequality is given by Stein using interpolation (see Theorem 1 in chapter 5 in [32]), but a more intrinsic proof using Young's inequality and radial symmetry follows by first applying symmetrization and then transferring the inequality to the multiplicative 
group $\mathbb{R}_{+}$. Once simply checks that the function

$$
\psi(t)=t^{n\left(\frac{1}{p}-\frac{1}{2}-\frac{\alpha}{2 n}\right)} \int_{S^{n-1}}\left[\left(t-\frac{1}{t}\right)^{2}+2\left(1-\xi_{1}\right)\right]^{-(n-\alpha) / 2} d \xi, \quad t>0
$$

is in $L^{r}\left(\mathbb{R}_{+}\right)$for $r=n /(n-\alpha)$. (See also the discussion of the Stein-Weiss theorem in the appendix to [10].) Observe that initial integration on the sphere is necessary for the application of Young's inequality.

Proof of Theorem 2. Apply the classical formula of Aronszajn-Smith (see 32] and Appendix to [11])

$$
\begin{gathered}
\int_{\mathbb{R}^{n} \times \mathbb{R}^{n}} \frac{\left|\left(\Lambda_{\alpha} f\right)(x)-\left(\Lambda_{\alpha} f\right)(y)\right|^{2}}{|x-y|^{n+2 \beta}} d x d y=D_{\beta} \int_{\mathbb{R}^{n}}|\xi|^{2 \alpha+2 \beta}|\hat{f}(\xi)|^{2} d \xi \\
D_{\beta}=\frac{2}{\beta} \pi^{\frac{n}{2}+2 \beta} \frac{\Gamma(1-\beta)}{\Gamma\left(\frac{n}{2}+\beta\right)}
\end{gathered}
$$

and use the dual form of the Hardy-Littlewood-Sobolev inequality due to Lieb:

$$
\int_{\mathbb{R}^{n}}|\xi|^{2 \alpha+2 \beta}|\hat{f}(\xi)|^{2} d \xi \geq c_{\alpha+\beta}\left[\|f\|_{L^{q^{*}\left(\mathbb{R}^{n}\right)}}\right]^{2}
$$

with

$$
c_{\alpha+\beta}=\pi^{-(\alpha+\beta)} \frac{\left(\frac{n}{2}+\alpha+\beta\right)}{\Gamma\left(\frac{n}{2}-\alpha+\beta\right)}\left[\frac{\Gamma\left(\frac{n}{2}\right)}{\Gamma(n)}\right]^{2(\alpha+\beta) / n}
$$

Putting these two statements together gives Theorem 2 ,

Corollary. For $f \in \mathcal{S}\left(\mathbb{R}^{n}\right), 0<\beta<1$ and $1 \leq p<n /(1+\beta)$

$$
\int_{\mathbb{R}^{n} \times \mathbb{R}^{n}} \frac{|(\nabla f)(x)-(\nabla f)(y)|^{p}}{|x-y|^{n+p \beta}} d x d y \geq c\left(\int_{\mathbb{R}^{n}}|f|^{q^{*}} d x\right)^{p / q^{*}}, \quad q^{*}=\frac{p n}{n-p(1+\beta)}
$$

Proof. Observe that by using the Aronszajn-Smith formula

$$
\int_{\mathbb{R}^{n} \times \mathbb{R}^{n}} \frac{|(\nabla f)(x)-(\nabla f)(y)|^{2}}{|x-y|^{n+p \beta}} d x d y=\int_{\mathbb{R}^{n} \times \mathbb{R}^{n}} \frac{\left|\left(\Lambda_{1} f\right)(x)-\left(\Lambda_{1} f\right)(y)\right|^{2}}{|x-y|^{n+p \beta}} d x d y
$$

and for $g=|\nabla f|$

$$
\int_{\mathbb{R}^{n} \times \mathbb{R}^{n}} \frac{|(\nabla f)(x)-(\nabla f)(y)|^{2}}{|x-y|^{n+p \beta}} d x d y \geq \int_{\mathbb{R}^{n} \times \mathbb{R}^{n}} \frac{\left|g^{*}(x)-g^{*}(y)\right|^{p}}{|x-y|^{n+p \beta}} d x d y
$$

Follow the steps in the proof of Theorem 1 where after Step 3 one has for $q=p n /(n-p \beta)$ and using the Sobolev inequality

$$
\left[\int_{\mathbb{R}^{n}}|\nabla f|^{q} d x\right]^{p / q} \geq c\left[\int_{\mathbb{R}^{n}}|f|^{q^{*}} d x\right]^{p / q^{*}}
$$

for $q^{*}=p n /(n-p(1+\beta))$. This gives the statement of the Corollary. 


\section{HAUSDORFF-Young INEQUALITIES FOR FRACTIONAL EMBEDDING}

The Aronszajn-Smith formula has proved to be highly useful:

$$
\int_{\mathbb{R}^{n} \times \mathbb{R}^{n}} \frac{|f(x)-f(y)|^{2}}{|x-y|^{n+2 \beta}} d x d y=D_{\beta} \int_{\mathbb{R}^{n}}|\xi|^{2 \beta}|\hat{f}(\xi)|^{2} d \xi
$$

By viewing this formula as an extension of the Plancherel theorem for fractional differentiation, one naturally looks to the role of the Hausdorff-Young inequality:

$$
\int_{\mathbb{R}^{n} \times \mathbb{R}^{n}} \frac{|f(x)-f(y)|^{p}}{|x-y|^{n+p \beta}} d x d y \rightsquigarrow \int_{\mathbb{R}^{n}}\left[|\xi|^{\beta}|| \hat{f}(\xi) \mid\right]^{p^{\prime}} d \xi
$$

where $p$ and $p^{\prime}$ are dual exponents.

Theorem 3. For $f \in \mathcal{S}\left(\mathbb{R}^{n}\right), 0<\beta<1,1<p<\infty$ and $1 / p+1 / p^{\prime}=1$

$$
\begin{aligned}
\int_{\mathbb{R}^{n} \times \mathbb{R}^{n}} \frac{|f(x)-f(y)|^{p}}{|x-y|^{n+p \beta}} d x d y & \geq c\left[\int_{\mathbb{R}^{n}}\left[|\xi|^{\beta}|\hat{f}(\xi)|\right]^{p^{\prime}} d \xi\right]^{p / p^{\prime}}, \quad 1<p \leq 2 \\
& \leq c\left[\int_{\mathbb{R}^{n}}\left[|\xi|^{\beta}|\hat{f}(\xi)|\right]^{p^{\prime}} d \xi\right]^{p / p^{\prime}}, \quad 2 \leq p<\infty
\end{aligned}
$$

Proof. Apply the Hausdorff-Young inequality for $1<p \leq 2$

$$
\begin{aligned}
& \int_{\mathbb{R}^{n} \times \mathbb{R}^{n}} \frac{|f(x)-f(y)|^{p}}{|x-y|^{n+p \beta}} d x d y=\int_{\mathbb{R}^{n}} \frac{1}{|w|^{n+p \beta}}\left[\int_{\mathbb{R}^{n}}|f(x+w)-f(x)|^{p} d x\right] d w \\
& \geq\left(c_{\mathrm{h}-\mathrm{y}}\right)^{p} \int_{\mathbb{R}^{n}} \frac{1}{|w|^{n+p \beta}}\left[\int_{\mathbb{R}^{n}}\left|e^{2 \pi i w \cdot \xi}-1\right|^{p^{\prime}}|\hat{f}(\xi)|^{p^{\prime}} d \xi\right]^{p / p^{\prime}} d w \\
& \geq\left(c_{\mathrm{h}-\mathrm{y}}\right)^{p}\left[\int_{\mathbb{R}^{n}}|\hat{f}(\xi)|^{p^{\prime}}\left[\int_{\mathbb{R}^{n}}\left|e^{2 \pi i w \cdot \xi}-1\right|^{p} \frac{1}{|w|^{n+p \beta}} d w\right]^{p^{\prime} / p} d \xi\right]^{p / p^{\prime}} \\
& \geq\left(c_{\mathrm{h}-\mathrm{y}}\right)^{p} \int_{\mathbb{R}^{n}}\left|e^{2 \pi i w \cdot \eta}-1\right|^{p} \frac{1}{|w|^{n+p \beta}} d w\left[\int_{\mathbb{R}^{n}}\left[|\xi|^{\beta}|\hat{f}(\xi)|\right]^{p^{\prime}} d \xi\right]^{p / p^{\prime}} \\
& =c\left[\int_{\mathbb{R}^{n}}\left[|\xi|^{\beta}|\hat{f}(\xi)|\right]^{p^{\prime}} d \xi\right]^{p / p^{\prime}}
\end{aligned}
$$

where $\eta \in S^{n-1}, c_{\mathrm{h}-\mathrm{y}}$ is the Hausdorff-Young constant and Minkowski's inequality for integrals was used to obtain the last inequality above. 
For $p \geq 2$ one just needs to reverse the string of inequalities:

$$
\begin{aligned}
\int_{\mathbb{R}^{n}} & \frac{1}{|w|^{n+p \beta}}\left[\int_{\mathbb{R}^{n}}|f(x+w)-f(x)|^{p} d x\right] d w \\
& \leq\left(c_{\mathrm{h}-\mathrm{y}}\right)^{p} \int_{\mathbb{R}^{n}} \frac{1}{|w|^{n+p \beta}}\left[\int_{\mathbb{R}^{n}}\left|e^{2 \pi i w \cdot \xi}-1\right|^{p^{\prime}}|\hat{f}(\xi)|^{p^{\prime}} d \xi\right]^{p / p^{\prime}} d w \\
& \leq\left(c_{\mathrm{h}-\mathrm{y}}\right)^{p}\left[\int_{\mathbb{R}^{n}}|\hat{f}(\xi)|^{p^{\prime}} d \xi\left[\int_{\mathbb{R}^{n}}\left|e^{2 \pi i w \cdot \xi}-1\right|^{p} \frac{1}{|w|^{n+p \beta}} d w\right]^{p^{\prime} / p} d \xi\right]^{p / p^{\prime}} \\
& =\left(c_{\mathrm{h}-\mathrm{y}}\right)^{p} \int_{\mathbb{R}^{n}}\left|e^{2 \pi i w \cdot \eta}-1\right|^{p} \frac{1}{|w|^{n+p \beta}} d w\left[\int_{\mathbb{R}^{n}}|\xi|^{\beta}|\hat{f}(\xi)|^{p^{\prime}} d \xi\right]^{p / p^{\prime}} \\
& =c\left[\int_{\mathbb{R}^{n}}\left[|\xi|^{\beta}|\hat{f}(\xi)|\right]^{p^{\prime}} d \xi\right]^{p / p^{\prime}}
\end{aligned}
$$

Here

$$
c_{\mathrm{h}-\mathrm{y}}=\left[p^{1 / p} / p^{\prime^{1 / p^{\prime}}}\right]^{-n / 2}
$$

\section{BiLineAR FRACTIONAL EMBEDDing}

Rigorous models for collision dynamics in plasmas suggest that bilinear fractional embedding estimates will be useful. Here the analysis developed above is applied to the forms:

$$
\int_{\mathbb{R}^{n} \times \mathbb{R}^{n}} \frac{|f(x) \nabla g(y)-f(y) \nabla g(x)|}{|x-y|^{n+\lambda}} d x d y \quad \rightsquigarrow \quad \int_{\mathbb{R}^{n} \times \mathbb{R}^{n}} \frac{|f(x) g(y)-f(y) g(x)|^{p}}{|x-y|^{n+\lambda}} d x d y
$$

As expected from classical Fourier analysis, these forms can be related to convolution formulas — both on the function side and on the Fourier transform side.

Theorem 4. For real-valued $f \in \mathcal{S}\left(\mathbb{R}^{n}\right), 0<\lambda<1$ and $\widetilde{f}(x)=f(-x)$

$$
\begin{gathered}
\int_{\mathbb{R}^{n} \times \mathbb{R}^{n}}|x-y|^{-n-\lambda}|f(x)(\nabla f)(y)-f(y)(\nabla f)(x)| d x d y \\
\geq 2 \int_{\mathbb{R}^{n}}|x|^{-n-\lambda}|\nabla(f * \widetilde{f})(x)| d x
\end{gathered}
$$

Proof.

$$
\begin{aligned}
\int_{\mathbb{R}^{n} \times \mathbb{R}^{n}}|x-y|^{-n-\lambda}|f(x)(\nabla f)(y)-f(y)(\nabla f)(x)| d x d y \\
=\int_{\mathbb{R}^{n} \times \mathbb{R}^{n}}|x|^{-n-\lambda}|f(x+y)(\nabla f)(y)-f(y)(\nabla f)(x+y)| d x d y \\
\geq \int_{\mathbb{R}^{n}}|x|^{-n-y} \mid \int_{\mathbb{R}^{n}}[f(x+y)(\nabla f(y)-f(y)(\nabla f)(x+y)] d y \mid d x \\
=\int_{\mathbb{R}^{n}}|x|^{-n-\lambda}\left|-2 \int_{\mathbb{R}^{n}}(\nabla f)(x+y) f(y) d y\right| d x \\
=2 \int_{\mathbb{R}^{n}}|x|^{-n-\lambda}|\nabla(f * \tilde{f})(x)| d x
\end{aligned}
$$


Observe that

$$
2 \nabla(f * \tilde{f})(0)=\int_{\mathbb{R}^{n}} \nabla\left(f^{2}\right) d y=0
$$

so the integrand above is locally integrable at $x=0$ for $0<\lambda<1$.

Theorem 5. For $f, g \in \mathcal{S}\left(\mathbb{R}^{n}\right), 1<p \leq 2,1 / p+1 / q=1$ and $0<\lambda<q$

$$
\begin{gathered}
\int_{\mathbb{R}^{n} \times \mathbb{R}^{n}}|x-y|^{-n-\lambda}|f(x) g(y)-f(y) g(x)|^{q} d x d y \\
\leq c\left[\int_{\mathbb{R}^{n}}\left|H_{\lambda / q}(u)\right|^{p} d u\right]^{q / p}
\end{gathered}
$$

where

$$
H_{\lambda / q}(u)=\int_{\mathbb{R}^{n}}|v|^{\lambda / q}\left|\widehat{f}\left(\frac{u+v}{2}\right) \widehat{g}\left(\frac{u-v}{2}\right)\right| d v .
$$

Proof.

$$
\begin{aligned}
\int_{\mathbb{R}^{n} \times \mathbb{R}^{n}}|x-y|^{-n-\lambda}|f(x) g(y)-f(y) g(x)|^{q} d x d y \\
=\int_{\mathbb{R}^{n} \times \mathbb{R}^{n}}|x|^{-n-\lambda}|f(x+y) g(y)-f(y) g(x+y)|^{q} d x d y \\
=\int_{\mathbb{R}^{n} \times \mathbb{R}^{n}}|x|^{-n-\lambda}\left|\int_{\mathbb{R}^{n} \times \mathbb{R}^{n}} e^{-2 \pi i y(\xi+n)}\left[e^{-2 \pi i x \xi}-e^{-2 \pi i x \eta}\right] \widehat{f}(\xi) \widehat{g}(\eta) d \xi d \eta\right|^{q} d x d y \\
=2^{-q} \int_{\mathbb{R}^{n} \times \mathbb{R}^{n}}|x|^{-n-\lambda}\left|\int_{\mathbb{R}^{n}} e^{-2 \pi i y u} \int_{\mathbb{R}^{n}} \Delta(x v) \widehat{f}\left(\frac{u+v}{2}\right) \widehat{g}\left(\frac{u-v}{2}\right) d u d v\right|^{q} d x d y
\end{aligned}
$$

where

$$
\Delta(x v)=e^{\pi i x v}-e^{-\pi i x v} .
$$

By applying the Hausdorff-Young inequality for $q \geq 2$, one obtains the upper bound

$$
\left(\frac{c_{\mathrm{h}-\mathrm{y}}}{2}\right)^{q} \int_{\mathbb{R}^{n}}|x|^{-n-\lambda}\left[\int_{\mathbb{R}^{n}}\left|\int_{\mathbb{R}^{n}}\right| \Delta(x v)|| \widehat{f}\left(\frac{u+v}{2}\right) \widehat{g}\left(\frac{u-v}{2}\right)|d v|^{p} d u\right]^{q / p} d x .
$$

The next objective is to interchange the $x$-integration with both integrals for $u$ and $v$ by using Minkowski's inequality for integrals twice. This gives

$$
\begin{aligned}
& \leq\left(\frac{c_{\mathrm{h}-\mathrm{y}}}{2}\right)^{q}\left[\int_{\mathbb{R}^{n}}\left[\int_{\mathbb{R}^{n}}|x|^{-n-\lambda}\left|\int_{\mathbb{R}^{n}}\right| \Delta(u v)|| \widehat{f}\left(\frac{u+v}{2}\right) \widehat{g}\left(\frac{u-v}{2}\right)|d v|^{q} d x\right]^{p / q} d u\right]^{q / p} \\
& \leq\left(\frac{c_{\mathrm{h}-\mathrm{y}}}{2}\right)^{q}\left[\int_{\mathbb{R}^{n}}\left[\int_{\mathbb{R}^{n}}\left(\int_{\mathbb{R}^{n}}|x|^{-n-\lambda}|\Delta(x v)|^{q} d x\right)^{1 / q}\left|\widehat{f}\left(\frac{u+v}{2}\right) \widehat{g}\left(\frac{u-v}{2}\right)\right| d v\right]^{p} d u\right]^{q / p} \\
& =c\left(\frac{c_{\mathrm{h}-\mathrm{y}}}{2}\right)^{q}\left[\int_{\mathbb{R}^{n}}\left[\int_{\mathbb{R}^{n}}|v|^{\lambda / q}\left|\widehat{f}\left(\frac{u+v}{2}\right) \widehat{g}\left(\frac{u-v}{2}\right)\right| d v\right]^{p} d u\right]^{q / p} \\
& =c\left(\frac{c_{\mathrm{h}-\mathrm{y}}}{2}\right)^{q}\left[\int_{\mathbb{R}^{n}}\left|H_{\lambda / q}(u)\right|^{p} d u\right]^{q / p}
\end{aligned}
$$


where $c_{\mathrm{h}-\mathrm{y}}$ is the Hausdorff-Young constant and

$$
c=\int_{\mathbb{R}^{n}}|x|^{-n-\lambda}|\Delta(x \cdot \eta)|^{q} d x, \quad \eta \in S^{n-1}
$$

where $0<\lambda<q$.

More careful analysis of the bilinear embedding integral for $p=2$ will give the analogue of the classical Aronszajn-Smith identity.

Theorem 6. For $f, g \in \mathcal{S}\left(\mathbb{R}^{n}\right)$ and $0<\lambda<2$

$$
\begin{aligned}
& \int_{\mathbb{R}^{n} \times \mathbb{R}^{n}}|x-y|^{-n-\lambda}|f(x) g(y)-f(y) g(x)|^{2} d x d y \\
& \quad=c \int_{\mathbb{R}^{n} \times \mathbb{R}^{n} \times \mathbb{R}^{n}}\left|v_{1}+v_{2}\right|^{\lambda} \widehat{f}\left(\frac{u+v_{1}}{2}\right) \widehat{g}\left(\frac{u-v_{1}}{2}\right)\left[\overline{\hat{f}}\left(\frac{u+v_{2}}{2}\right) \hat{\widehat{g}}\left(\frac{u-v_{2}}{2}\right)-\overline{\hat{f}}\left(\frac{u-v_{2}}{2}\right) \overline{\widehat{g}}\left(\frac{u+v_{2}}{2}\right)\right] d u d v_{1} d v_{2}
\end{aligned}
$$

where

$$
c=\left(\frac{\pi}{2}\right)^{\lambda} \frac{\pi^{n / 2}}{\lambda} \frac{\Gamma\left(1-\frac{\lambda}{2}\right)}{\Gamma\left(\frac{n+\lambda}{2}\right)} .
$$

Here the value of the integral

$$
\int_{\mathbb{R}^{n}} \frac{1}{|w|^{n+\lambda}}(1-\cos w \cdot \eta) d w=\frac{2^{1-\lambda} \pi^{n / 2}}{\lambda} \frac{\Gamma\left(1-\frac{\lambda}{2}\right)}{\Gamma\left(\frac{n+\lambda}{2}\right)}
$$

was calculated in the appendix of [11].

Functional product decomposition coupled with fractional embedding norms modeled after plasma collision dynamics can be used to determine new inequalities of Bourgain-BrezisMironceau type.

Theorem 7. For $f, g \in \mathcal{S}\left(\mathbb{R}^{n}\right), 0<\beta<1$ and $1 \leq p<n / \beta$

$$
\begin{aligned}
& \int_{\mathbb{R}^{n} \times \mathbb{R}^{n} \times \mathbb{R}^{n} \times \mathbb{R}^{n}}\left[(x-u)^{2}+(y-v)^{2}\right]^{-(2 n+p \beta) / 2}|f(x) g(y)-f(u) g(v)|^{p} d x d y d u d v \\
& \geq c\left\{\begin{array}{l}
{\left[\|f\|_{L^{p}\left(\mathbb{R}^{n}\right)}\|g\|_{L^{q}\left(\mathbb{R}^{n}\right)}\right]^{p}} \\
{\left[\|g\|_{L^{p}\left(\mathbb{R}^{n}\right)}\|f\|_{L^{q}\left(\mathbb{R}^{n}\right)}\right]^{p}}
\end{array}\right.
\end{aligned}
$$

where $q=p n /(n-p \beta)$. For $p=2$, the sharp value of the constant is given by

$$
c=\frac{2 \pi^{\beta+n}}{\beta} \frac{\Gamma(1-\beta)}{\Gamma\left(\frac{n}{2}-\beta\right)} \frac{\Gamma\left(\frac{n}{2}+\beta\right)}{\Gamma(n+\beta)}\left[\frac{\Gamma\left(\frac{n}{2}\right)}{\Gamma(n)}\right]^{2 \beta / n}
$$

Proof. By applying the Symmetrization Lemma separately in $(x, u)$ and $(y, v)$, the integral is reduced by using the equimeasurable radial decreasing rearrangements of $f$ and $g$. Then apply the triangle inequality lemma for the function $f$ to obtain the lower bound:

$$
\begin{gathered}
\int_{\mathbb{R}^{n}}|f|^{p} d x \int_{\mathbb{R}^{n}}\left(1+|x|^{2}\right)^{-(2 n+p \beta) / 2} d x \int_{\mathbb{R}^{n} \times \mathbb{R}^{n}}|y-v|^{-n-p \beta}\left|g^{*}(y)-g^{*}(v)\right|^{p} d y d v \\
\geq c \int_{\mathbb{R}^{n}}|f|^{p} d x\left(\int_{\mathbb{R}^{n}}|g|^{q} d x\right)^{p / q}, \quad q=\frac{p n}{n-p \beta} .
\end{gathered}
$$


Since the fractional embedding norm is symmetric in the role of the functions $f$ and $g$, this gives equation (24). Observe that in fact the finiteness of the embedding norm implies that $f, g \in L^{p}\left(\mathbb{R}^{n}\right) \cap L^{q}\left(\mathbb{R}^{n}\right)$. The sharp value of the constant for $p=2$ can be obtained using the Aronszajn-Smith formula for $\mathbb{R}^{2 n}$ and then applying Theorem 2 for $\alpha=0$.

While this analysis is instructive and perhaps useful in understanding collision dynamics, it does reflect that restriction to product functions will not accurately show the appropriate control by multilinear embedding (see discussion on page 185 in [12]).

\section{Fractional smoothing on the Heisenberg group}

The Heisenberg group is a natural setting to study problems with mixed homogeneity. As a nilpotent Lie group, it gives the simplest extension of $\mathbb{R}^{n}$ where homogeneity is broken but a substantive part of the Euclidean translation group action is retained. Moreover the group possesses an $S L(2, \mathbb{R})$ symmetry associated with its dilation structure which brings into play the non-unimodular group associated with the hyperbolic plane. The complexity of the symmetry structure makes some questions arising from Euclidean analysis hard while other issues become relatively simple to sort out with multiple approaches. This framework highlights the intrinsic tension between viewing a nilpotent semisimple group arising from complex geometry versus a manifold with non-positive curvature and a non-unimodular group action as the most characteristic extension of $\mathbb{R}^{n}$.

The Heisenberg group $\mathcal{H}_{n}$ is realized as the boundary of the Siegel upper half-space in $\mathbb{C}^{n+1}$, $\left.D=\int z \in \mathbb{C}^{n+1}: \operatorname{Im} z_{n+1}>\left|z_{1}\right|^{2}+\cdots+\left|z_{n}\right|^{2}\right\}$. Then

$$
\mathcal{H}_{n}=\left\{w=(z, t): z \in \mathbb{C}^{n}, t \in \mathbb{R}\right\}
$$

with the group action

$$
w w^{\prime}=(z, t)\left(z^{\prime}, t^{\prime}\right)=\left(z+z^{\prime}, t+t^{\prime}+2 \operatorname{Im} z \bar{z}^{\prime}\right)
$$

and Haar measure on the group is given by

$$
d w=d z d \bar{z} d t=4^{n} d x d y d t
$$

where $z=x+i y \in \mathbb{C}^{\prime \prime}$ and $t \in \mathbb{R}$. The natural metric is

$$
d\left(w, w^{\prime}\right)=d\left((z, t),\left(z^{\prime}, t^{\prime}\right)\right)=d\left(w^{\prime}-1 w, \widehat{0}\right)
$$

with

$$
d(w, \widehat{0})=d((z, t),(0,0))=\left.|| z\right|^{2}+\left.i t\right|^{1 / 2}=\left.|| z\right|^{4}+\left.t^{2}\right|^{1 / 4}=|w| .
$$

To illustrate the relation with fractional smoothness, two examples expanding on Besov norms and Stein-Weiss fractional integrals are developed here. But first as a technical tool to facilitate application relative to problems with mixed homogeneity, the "triangle inequality" lemma is rephrased as a convolution inequality.

Lemma 3. For $k \geq 0$ and $f, g, k$ satisfying suitable integrability conditions

$$
\begin{aligned}
& \int k(u, v, t-s)|f(u, t)-g(v, s)|^{p} d u d v d s d t \\
& \quad \geq \int\left[\int k(u, v, t) d t\right]|F(u)-G(v)|^{p} d u d v
\end{aligned}
$$

where

$$
F(u)=\left(\int|f(u, t)|^{p} d t\right)^{1 / p} \quad, \quad G(v)=\left(\int|g(v, t)|^{p} d t\right)^{1 / p}
$$


Proof. Observe that

$$
\begin{aligned}
& \int k(u, v, t-s)|f(u, t)-g(v, s)|^{p} d u d v d t d s \\
& \quad=\int k(u, v, t)\left[\int|f(u, t+s)-g(v, s)|^{p} d s\right] d u d v d t
\end{aligned}
$$

and apply the "triangle inequality" for the $s$-integration; that is

$$
\int|f(u, t+s)-g(v, s)|^{p} d s \geq|F(u)-G(v)|^{p} .
$$

Theorem 8. Let $f \in \mathcal{S}\left(\mathcal{H}_{n}\right), 0<\beta<1$ and $1 \leq p<2 n / \beta$; then

$$
\begin{gathered}
\int_{\mathcal{H}_{n} \times \mathcal{H}_{n}} \frac{\left|f(w)-f\left(w^{\prime}\right)\right|^{p}}{\left[d\left(w, w^{\prime}\right)\right]^{2 n+2+p \beta}} d w d w^{\prime} \geq F_{p, \beta} \int_{\mathcal{H}_{n}}|z|^{-p \beta}|f|^{p} d w \\
F_{p, \beta}=\left.\left.\frac{4^{n} \sqrt{\pi} \Gamma\left[\frac{2 n+p \beta}{4}\right]}{\Gamma\left[\frac{2 n+2+p \beta}{4}\right]} \int_{\mathbb{R}^{2 n}}|1-| x\right|^{-\lambda}\right|^{p}|x-\eta|^{2 n-p \beta} d x
\end{gathered}
$$

for $\lambda=(2 n-p \beta) / p$ and $\eta \in S^{2 n-1}$.

Proof. Apply the "triangle inequality" to the $t, t^{\prime}$ integrations

$$
\begin{aligned}
\int_{\mathcal{H}_{n} \times \mathcal{H}_{n}} & \frac{\left|f(w)-f\left(w^{\prime}\right)\right|^{p}}{\left[d\left(w, w^{\prime}\right)\right]^{2 n+2+p \beta}} d w d w^{\prime} \\
\geq & \int_{\mathbb{C}^{n} \times \mathbb{C}^{n}}\left[\int_{\mathbb{R}}\left[\left|z-z^{\prime}\right|^{4}+t^{2}\right]^{-(2 n+2+p \beta) / 4} d t\right]\left|h(z)-h\left(z^{\prime}\right)\right|^{p} d z d \bar{z} d z^{\prime} d \bar{z}^{\prime} \\
= & \frac{\sqrt{\pi} \Gamma\left[\frac{2 n+p \beta}{4}\right]}{\Gamma\left[\frac{2 n+2+p \beta}{4}\right]} \int_{\mathbb{C}^{n} \times \mathbb{C}^{n}} \frac{\left|h(z)-h\left(z^{\prime}\right)\right|^{p}}{\left|z-z^{\prime}\right|^{2 n+p \beta}} d z d \bar{z} d z^{\prime} d \bar{z}^{\prime} \\
\geq & \left.\left.\frac{4^{n} \sqrt{\pi} \Gamma\left[\frac{2 n+p \beta}{4}\right]}{\Gamma\left[\frac{2 n+2+p \beta}{4}\right]} \int_{\mathbb{R}^{2 n}}|1-| x\right|^{-\lambda}\right|^{p}|x-\eta|^{2 n-p \beta} d x \int_{\mathbb{C}^{n}}|z|^{-p \beta}|h|^{p} d z d \bar{z}
\end{aligned}
$$

for $\lambda=(2 n-p \beta) / p$ and $\eta \in S^{2 n-1}$ and using Lemma 1 with

$$
h(z)=\left(\int_{\mathbb{R}}|f(z, t)|^{p} d t\right)^{1 / p}
$$

to obtain inequality (26) above.

By combining methods taken from earlier papers (see Theorem 3 in [8, Theorem 5 in [10]), one can obtain sharp estimates for Stein-Weiss fractional integrals on the Heisenberg group as maps from $L^{p}\left(\mathcal{H}_{n}\right)$ to $L^{p}\left(\mathcal{H}_{n}\right)$ for $1<p<\infty$ (see also [23] where different arguments are developed for the analysis of Stein-Weiss integrals). 
Theorem 9. For $f \in L^{p}\left(\mathcal{H}_{n}\right), 1<p<\infty, w=(z, t) \in \mathcal{H}_{n}, 0<\lambda<2 n+2, \alpha<2 n / p$, $\beta<2 n / p^{\prime}, 1 / p+1 / p^{\prime}=1$ and $2 n+2=\lambda+\alpha+\beta$

$$
\begin{gathered}
\left\||z|^{-\alpha}\left(|w|^{-\lambda} *\left(|z|^{-\beta} f\right)\right)\right\|_{L^{p}\left(\mathcal{H}_{n}\right)} \leq D_{\alpha, \beta}\|f\|_{L^{p}\left(\mathcal{H}_{n}\right)} \\
D_{\alpha, \beta}=\left(4 \pi^{2}\right)^{n} \sqrt{\pi}\left[\frac{\Gamma\left[\frac{2 n-\alpha-\beta}{4}\right] \Gamma\left[\frac{\alpha+\beta}{2}\right] \Gamma\left[\frac{n}{p}-\frac{\alpha}{2}\right] \Gamma\left[\frac{n}{p^{\prime}}-\frac{\beta}{2}\right]}{\Gamma\left[\frac{2 n+2-\alpha-\beta}{4}\right] \Gamma\left[\frac{2 n-\alpha-\beta}{2}\right] \Gamma\left[\frac{n}{p^{\prime}}+\frac{\alpha}{2}\right] \Gamma\left[\frac{n}{p}+\frac{\beta}{2}\right]}\right]
\end{gathered}
$$

Proof. Apply the sharp $L^{1}$ Young's inequality for convolution in the $t$-variable which will then result in a reduction to the corresponding Stein-Weiss fractional integral on $\mathbb{R}^{2 n}$ (see Theorem 2 in [10]). Here $|w|$ denotes $\left(|z|^{4}+t^{2}\right)^{1 / 4}$ which defines the metric on $\mathcal{H}_{n}$, and $*$ denotes convolution on the group in equation (27).

$$
\left\||z|^{-\alpha}\left(|w|^{-\lambda} *\left(|z|^{-\beta} f\right)\right)\right\|_{L^{p}\left(\mathcal{H}_{n}\right)} \leq\left\||z|^{-\alpha}\left(J *\left(|z|^{-\beta} h\right)\right)\right\|_{L^{p}\left(\mathbb{C}^{n}\right)}
$$

where

$$
\begin{gathered}
h(z)=\left[\int_{\mathbb{R}}|f(z, t)|^{p} d t\right]^{1 / p}, \quad\|h\|_{L^{p}\left(\mathbb{C}^{n}\right)}=\|f\|_{L^{p}\left(\mathcal{H}_{n}\right)}, \\
J(z)=\int_{\mathbb{R}}\left(|z|^{4}+t^{2}\right)^{-\lambda / 4} d t=|z|^{-\lambda+2} \int_{-\infty}^{\infty}\left(1+t^{2}\right)^{-\lambda / 4} d t \\
=\frac{\sqrt{\pi} \Gamma\left(\frac{2 n-\alpha-\beta}{4}\right)}{\Gamma\left(\frac{2 n+2-\alpha-\beta}{4}\right)}|z|^{-\lambda+2}
\end{gathered}
$$

then

$$
\begin{aligned}
& \left\||z|^{-\alpha}\left(J *\left(|z|^{-\beta} h\right)\right)\right\|_{L^{p}\left(\mathbb{C}^{n}\right)} \\
& \quad=4^{n\left(1+\frac{1}{p}\right)} \sqrt{\pi} \frac{\Gamma\left[\frac{2 n-\alpha-\beta}{4}\right]}{\Gamma\left[\frac{2 n+2-\alpha-\beta}{4}\right]}\left\||x|^{-\alpha}\left(|x|^{-\alpha} *\left(|x|^{-\beta} h\right)\right)\right\|_{L^{p}\left(\mathbb{R}^{2 n}\right)} \\
& \quad \leq\left(4 \pi^{2}\right)^{n} \sqrt{\pi}\left[\frac{\Gamma\left[\frac{2 n-\alpha-\beta}{4}\right] \Gamma\left[\frac{\alpha+\beta}{2}\right] \Gamma\left[\frac{n}{p}-\frac{\alpha}{2}\right] \Gamma\left[\frac{n}{p^{\prime}}-\frac{\beta}{2}\right]}{\Gamma\left[\frac{2 n+2-\alpha-\beta}{4}\right] \Gamma\left[\frac{2 n-\alpha-\beta}{2}\right] \Gamma\left[\frac{n}{p^{\prime}}+\frac{\alpha}{2}\right] \Gamma\left[\frac{n}{p}+\frac{\beta}{2}\right]}\right]\|h\|_{L^{p}\left(\mathbb{C}^{n}\right)} \\
& \quad=D_{\alpha, \beta}\|f\|_{L^{p}\left(\mathcal{H}_{n}\right)}
\end{aligned}
$$

Notice that as demonstrated by the above calculation the hypothesis requires that $\lambda>2$ so that clearly the integral above for $J$ is well-defined.

Analysis on the Heisenberg group reflects the characteristic property that the transitive group action "breaks" the closely coupled Euclidean translation and homogeneity in a way that does not allow intrinsic symmetries to clearly determine precise estimates. The complexity of the intertwining of lower-order invariance on the Heisenberg group, perhaps reflecting the influence of complex geometry, does not facilitate decoupling the contrasting symmetries. Partial discussion of this behavior is given in arguments contained in the author's paper [6]. Proofs for the theorems above use integrability for the kernel to simply remove the non-Euclidean part of the convolution. But here a reductioin to functions radial in the $z$ variable will provide alternate proofs that utilize the underlying two-dimensional dilation symmetry on $\mathcal{H}_{n}$ that corresponds 
to the embedded action of $S L(2, \mathbb{R})$ and hyperbolic space $\mathbb{H}^{2}$. Start with the metric on the Heisenberg group,

$$
\begin{aligned}
& \left|w^{\prime}-1 w\right|=\left[\left|z-z^{\prime}\right|^{4}+\left|t-t^{\prime}-2 \operatorname{Im} z \bar{z}^{\prime}\right|^{2}\right]^{1 / 4} \\
& =\left[\left.|| z\right|^{2}+\left|z^{\prime}\right|^{2}-\left.2 \operatorname{Re} z \bar{z}^{\prime}\right|^{2}+\left|t-t^{\prime}-2 \operatorname{Im} z \bar{z}^{\prime}\right|^{2}\right]^{1 / 4} ;
\end{aligned}
$$

without too much overlap, change notation so that $y=|z|$ and $u_{z} \in S U(n)$ with $u_{z}(1,0)=z /|z|$, $\langle u\rangle=u_{1,1}$; then

$$
\begin{aligned}
\left|w^{\prime-1} w\right| & =\left[\left|y+y^{\prime}-2 \sqrt{y y^{\prime}} \operatorname{Re}\left\langle U^{\prime}-1 U\right\rangle\right|^{2}+\left|t-t^{\prime}-2 \sqrt{y y^{\prime}} \operatorname{Im}\left\langle U^{\prime}-1 U\right\rangle\right|^{2}\right]^{1 / 4} \\
& =\left(4 y y^{\prime}\right)^{1 / 4}\left[\left|\frac{y+y^{\prime}}{2 \sqrt{y y^{\prime}}}-\operatorname{Re} \zeta\right|^{2}+\left|\frac{t-t^{\prime}}{2 \sqrt{y y^{\prime}}}-\operatorname{Im} \zeta\right|^{2}\right]^{1 / 4}, \quad \zeta=\left\langle U^{\prime}-1 U^{1 / 4}\right\rangle \\
& =\left(4 y y^{\prime}\right)^{1 / 4}\left[\rho^{2}-2 \rho|\zeta| \cos (\theta-\varphi)+|\zeta|^{2}\right]^{1 / 4}
\end{aligned}
$$

where

$$
\rho^{2}=\left(\frac{y+y^{\prime}}{2 \sqrt{y y^{\prime}}}\right)^{2}+\left(\frac{t-t^{\prime}}{2 \sqrt{y y^{\prime}}}\right)^{2}=1+\delta^{2}
$$

with $\delta$ denoting the Poincaré metric on $\mathbb{H}^{2}$

$$
\delta=\frac{1}{2 \sqrt{y y^{\prime}}} \sqrt{\left(t-t^{\prime}\right)^{2}+\left(y-y^{\prime}\right)^{2}}=\operatorname{dist}\left[(t, y),\left(t^{\prime}, y^{\prime}\right)\right]
$$

and

$$
\tan \theta=\frac{t-t^{\prime}}{y+y^{\prime}}, \quad \frac{-\pi}{2}<\theta<\frac{\pi}{2} ; \quad \zeta=|\zeta| e^{i \varphi}, \quad 0 \leq \varphi<2 \pi .
$$

the connected rotations defined by the angles $\theta$ and $\varphi$, one depending on the dilation variables and the other on the variables coming from the complex boundary of the ball, create the difficulty for reduction to lower-dimensional submanifolds. But the problems here allow an integration to remove this interaction so that the kernel depends only on the Poincaré metric as a decreasing function of $\rho$ or $\delta$ :

$$
\psi_{\lambda}(\rho)=\int_{\partial B_{n}}\left|\rho-\zeta_{1}\right|^{-\lambda} d \zeta
$$

where $\partial B_{n}=\left\{\zeta \in \mathbb{C}^{n}:|\zeta|^{2}=\sum\left|\zeta_{k}\right|^{2}=1\right\}$ and $d \zeta$ denotes normalized surface measure on the boundary. This representation allows not only development of an alternate framework for the proofs of Theorems 8 and 9 but gives new insight on the role of embedding and convolution estimates on hyperbolic space $\mathbb{H}^{n}$ and non-unimodular groups with non-positive curvature.

Alternate proof (Theorem 9). The weighted fractional integral on the Heisenberg group given by equation (27) is equivalent to the inequality on $\mathbb{H}^{2}$

$$
\begin{gathered}
\left|\int_{\mathbb{H}^{2} \times \mathbb{H}^{2}} G(v) K\left(v^{\prime}-1 v\right) F\left(v^{\prime}\right) d \nu d \nu^{\prime}\right| \leq C_{\alpha, \beta, p}\|F\|_{L^{p}\left(\mathbb{H}^{2}\right)}\|G\|_{L^{p^{\prime}\left(\mathbb{H}^{2}\right)}} \\
\|F * K\|_{L^{p}\left(\mathbb{H}^{2}\right)} \leq C_{\alpha, \beta, p}\|F\|_{L^{p}\left(\mathbb{H}^{2}\right)} \\
C_{\alpha, \beta, p}=\left\|\Delta^{-1 / p^{\prime}} K\right\|_{L^{1}\left(\mathbb{H}^{2}\right)}
\end{gathered}
$$

where

$$
K(v)=y^{\sigma} \psi_{\lambda}[\rho(v, \hat{0})]
$$


with $\sigma=(n+1)(1 / p-1 / 2)-\alpha / 4+\beta / 4, \rho^{2}=1+\delta^{2}(v, \hat{0}), \lambda=2 n+2-\alpha-\beta$, and $\Delta$ denotes the modular function on $\mathbb{H}^{2}$. Here Young's inequality for non-unimodular groups is applied. As for the Euclidean case, the $L^{1}$ estimate is sharp.

Alternate proof (Theorem 8). First apply the triangle inequality in the angular variables on $S^{2 n-1}$ to reduce the problem to functions radial in $z$ and the integration falls over the variables $(|z|, t)$.

$$
\begin{aligned}
& \int_{\mathcal{H}_{n} \times \mathcal{H}_{n}} \frac{\left|f(w)-f\left(w^{\prime}\right)\right|^{p}}{\left[d\left(w, w^{\prime}\right)\right]^{2 n+2+p \beta}} d w d w^{\prime} \\
& \quad \geq \int_{\mathcal{H}_{n} \times \mathcal{H}_{n}}\left|F(|z|, t)-F\left(\left|z^{\prime}\right|, t^{\prime}\right)\right|^{p}\left[\int\left[d\left(w, w^{\prime}\right)\right]^{-(2 n+2+p \beta)} d \hat{\xi}\right] d w d w^{\prime}
\end{aligned}
$$

where $d w=d z d \bar{z} d t=4^{n} r^{2 n-1} d r d \xi d t$ with $d \hat{\xi}$ denoting normalized surface measure on $S^{2 n-1}$ and

$$
F(|z|, t)=\left[\int_{S^{2 n-1}}|f(w)| d \hat{\xi}\right]
$$

To repose this problem on the hyperbolic plane $\mathbb{H}^{2}$, set $y=|z|^{2}$. Then

$$
\begin{aligned}
& \int_{\mathcal{H}_{n} \times \mathcal{H}_{n}}\left|F(|z|, t)-F\left(\left|z^{\prime}\right|, t^{\prime}\right)\right|^{p}\left[\int_{S^{2 n-1}}\left[d\left(w, w^{\prime}\right)\right]^{-(2 n+2+p \beta)} d \hat{\xi}\right] d w d w^{\prime} \\
& =(4 \pi)^{2 n} 2^{-(n+1+p \beta / 2)} /[\Gamma(n)]^{2} \int_{\mathbb{H}^{2} \times \mathbb{H}^{2}} \psi_{\lambda}(\rho)\left|h(v)\left(y^{\prime} / y\right)^{\sigma / 2}-h\left(v^{\prime}\right)\left(y / y^{\prime}\right)^{\sigma / 2}\right|^{p} d \nu d \nu^{\prime}
\end{aligned}
$$

where $d \nu=y^{-2} d x d y$ is Haar measure on $\mathbb{H}^{2}, \sigma=\frac{n+1}{p}-\frac{\beta}{2}$ and

$$
\psi_{\lambda}(\rho)=\int_{\partial B_{n}}\left|\rho-\zeta_{1}\right|^{-\lambda} d \zeta
$$

where $\lambda=2 n+2+p \beta$ and $\rho=\sqrt{1+\delta^{2}}, \delta=d\left(v, v^{\prime}\right)$ is the Poincaré metric on $\mathbb{H}^{2}$ with $v=(x, y) \in \mathbb{H}^{2} . \quad h(v)=F(\sqrt{y}, x) y^{-(n+1-p \beta) / p}$ and the one-dimensional variable $t$ has be re-labeled as $x$. Further setting

$$
g(v)=y^{\sigma / 2}\left[\psi_{\lambda}(\rho)\right]^{1 / p}
$$

where here $\rho=\sqrt{1+\operatorname{dist}(v, \hat{0})^{2}}$ with $\hat{0}=(0,1)$ being the origin in $\mathbb{H}^{2}$.

Then

$$
\begin{gathered}
\int_{\mathbb{H}^{2} \times \mathbb{H}^{2}} \psi_{\lambda}(\rho)\left|h(v)\left(y^{\prime} / y\right)^{\sigma / 2}-h\left(v^{\prime}\right)\left(y / y^{\prime}\right)^{\sigma / 2}\right|^{p} d \nu d \nu^{\prime} \\
=\int_{\mathbb{H}^{2} \times \mathbb{H}^{2}}\left|g\left(v^{-1} v^{\prime}\right) h(v)-g\left(v^{\prime-1} v\right) h\left(v^{\prime}\right)\right|^{p} d \nu d \nu^{\prime} \\
\geq C_{p, \beta} \int_{\mathbb{H}^{2}}|h|^{p} d \nu
\end{gathered}
$$

by applying the triangle inequality for non-unimodular groups (see equation (4.3) on page 189 in [11]) where

$$
C_{p, \beta}=\int_{\mathbb{H}^{2}}|| g(v)\left|-\Delta(v)^{-1 / p}\right| g\left(v^{-1}\right)||^{p} d \nu
$$

with $\Delta$ denoting the modular function which is $1 / y$ on $\mathbb{H}^{2}$. Checking all the changes in variables results in the constant obtained in equation (26) above. 
For clarity, the basic inequalities for a Lie group are listed in the following lemma:

Convolution Lemma. Let $G$ be a locally compact group with left-invariant Haar measure denoted by $m$. For $1 \leq p \leq \infty$

$$
\begin{gathered}
\|f * g\|_{L^{p}(G)} \leq\|f\|_{L^{p}(G)}\left\|\Delta^{-1 / p^{\prime}} g\right\|_{L^{1}(G)} \\
\|f * g\|_{L^{p}(G)} \leq\|f\|_{L^{1}(G)}\|g\|_{L^{p}(G)} \\
\|f * g\|_{L^{r}(G)} \leq\|f\|_{L^{p}(G)}\left\|\Delta^{-1 / p^{\prime}} g\right\|_{L^{q}(G)}
\end{gathered}
$$

where $\Delta$ denotes the modular function defined by $m(E y)=\Delta(y) m(E), 1 / p+1 / p^{\prime}=1$ and $1 / r=1 / p+1 / q-1$. For $f, g, h \in L^{p}(G), 1 \leq p<\infty$

$$
\begin{aligned}
\int_{G \times G} & \left|g\left(x^{-1} y\right) f(x)-h\left(y^{-1} x\right) f(y)\right|^{p} d m d m \\
& \geq \int_{G}|| g(y)\left|-\Delta(y)^{-1 / p}\right| h\left(y^{-1}\right)||^{p} d m \int_{G}|f(x)|^{p} d m
\end{aligned}
$$

The first, second and fourth inequalities are optimal.

\section{PitT'S Inequality}

The Hausdorff-Young estimates obtained above allow one to give some reasonable (though still not optimal) constants for Pitt's inequality on the line of duality. From the Appendix in [10]:

Pitt's Inequality. For $f \in \mathcal{S}\left(\mathbb{R}^{n}\right), 1<p \leq q<\infty, 0<\alpha<n / q, 0<\beta<n / p^{\prime}$ and $n \geq 2$

$$
\left[\left.\left.\int_{\mathbb{R}^{n}}|| x\right|^{-\alpha} \widehat{f}\right|^{q} d x\right]^{1 / q} \leq A\left[\left.\left.\int_{\mathbb{R}^{n}}|| x\right|^{\beta} f\right|^{p} d x\right]^{1 / p}
$$

with the index constraint

$$
\frac{n}{p}+\frac{n}{q}+\beta-\alpha=n
$$

For $\alpha=\beta, p$ and $q$ are dual exponents. Changing notation in equation (20), one now has the following form of Pitt's inequality:

Theorem 10. For $f \in \mathcal{S}\left(\mathbb{R}^{n}\right)$ with $0<\beta<1,1<p \leq 2$ and $\frac{1}{p}+\frac{1}{p^{\prime}}=1$

$$
\begin{gathered}
{\left[\left.\left.\int_{\mathbb{R}^{n}}|| x\right|^{-\beta} \widehat{f}\right|^{p^{\prime}} d x\right]^{1 / p^{\prime}} \leq A\left[\left.\left.\int_{\mathbb{R}^{n}}|| x\right|^{\beta} f\right|^{p} d x\right]^{1 / p}} \\
A=\left[p^{1 / p} / p^{1 / p^{\prime}}\right]^{n / 2} \frac{\left[\int_{\mathbb{R}^{n}}\left|e^{2 \pi i w \cdot \eta}-1\right|^{p^{\prime}} \frac{1}{|w|^{n+p^{\prime} \beta}} d w\right]^{1 / p^{\prime}}}{\left[\left.\int_{\mathbb{R}^{n}}|1-| x\right|^{-\lambda}\left|p^{\prime}\right| x-\left.\eta\right|^{-n-p^{\prime} \beta} d x\right]^{1 / p^{\prime}}}
\end{gathered}
$$

with $\lambda=\left(n-p^{\prime} \beta\right) / p^{\prime}$.

Pitt's inequality is a natural expression of the uncertainty principle - to measure the balance between the relative size of a function and its Fourier transform at infinity. This underlying structure was outlined in an earlier paper [7. Not only does Pitt's inequality determine uncertainty, but it offers insight into the nature of optimal constants and provides an asymptotic upper bound for the constant given by the extended uncertainty inequality:

$$
\left[\int_{\mathbb{R}^{n}}|f|^{2} d x\right]^{2} \leq B_{\alpha} \int_{\mathbb{R}^{n}}|x|^{\alpha}|f|^{2} d x \int_{\mathbb{R}^{n}}|\xi|^{\alpha}|\widehat{f}|^{2} d \xi
$$


with $B_{\alpha} \lesssim(4 \pi / n)^{\alpha}$ for $\alpha>0$. Observe that if the non-optimal constant taken from Pitt's inequality is used for $B_{\alpha}$

$$
\pi^{\alpha}\left[\Gamma\left(\frac{n-\alpha}{4}\right) / \Gamma\left(\frac{n+\alpha}{4}\right)\right]^{2}
$$

then one obtains the sharp logarithmic uncertainty inequality in the limit $\alpha \rightarrow 0$.

\section{ApPendix: Proof of the SyMmetrization LEMma}

The "two-point" inequality lies at the heart of many results on rearrangement and symmetrization echoing a central spirit from the work of Hardy and Littlewood. Here the simplest form is given by the numerical inequality for sets of distinct positive real numbers $\left\{a_{1}, a_{2}\right\}$, $\left\{b_{1}, b_{2}\right\}$ with

$$
a_{1} b_{2}+a_{2} b_{2} \leq a^{*} b^{*}+a_{*} b_{*}
$$

where $c^{*}=\max \left\{c_{1}, c_{2}\right\}$ and $c_{*}=\min \left\{c_{1}, c_{2}\right\}$. Extending to finite sequences $\left\{c_{k}\right\}$ with $\left\{c_{k}^{*}\right\}$ given by sequential rearrangement in terms of decreasing size

$$
\sum_{1}^{N} a_{k} b_{k} \leq \sum_{1}^{N} a_{k}^{*} b_{k}^{*} .
$$

The Symmetrization Lemma used in the argument for Theorem 1 above corresponds to a two-function rearrangement inequality and its proof depends on two simple ideas which are outwardly independent of the geometric character of Lebesgue measure:

(1) symmetrization of functions or sets can be achieved as the limit of a sequence of lowerdimensional symmetrizations;

(2) rearrangement inequalities involving only two functions can often be obtained from symmetrization on two points.

The argument depends on having a manifold with a reflection symmetry that divides the space into two equivalent half-spaces with the property that for two points $P, Q$ in the same half-space then

$$
\operatorname{dist}(P, Q) \leq \operatorname{dist}(P, \tilde{Q})
$$

where $\tilde{Q}$ is the reflection of $Q$ into the other half-space. In addition, one needs a transitive group action that "moves points on the manifold around".

Two-Point Symmetrization Lemma. Consider a $\sigma$-finite measure space $M$ invariant under an involution symmetry $\sigma$. Suppose $M$ has a mutually disjoint decomposition $M=M_{+} \cup M-\cup P$ with $P=\{x \in M: \sigma(x)=x\}$ being a set of measure zero, $M_{-}=\sigma\left(M_{+}\right)$and $d(x, y) \leq$ $d(x, \sigma(y))$ for $s, y \in M_{+} . k$ and $\rho$ are two nonnegative functions defined on $M \times M$ with

(i) $k[\sigma(x), \sigma(y)]=k(x, y), \rho[\sigma(x), \sigma(y)]=\rho(x, y)$

(ii) $k(x, y) \geq k[x, \sigma(y)], \rho(x, y) \leq \rho[x, \sigma(y)]$ for $x, y \in M_{+}$.

$\varphi$ is a nonnegative function defined on $[0, \infty)$ with the following properties: $\varphi(0)=0, \varphi$ convex and monotone increasing, $\varphi^{\prime \prime}(0) \geq 0$ and $f \varphi^{\prime}(t)$ convex. Define $f^{*}(x)=\max \{f(x), f[\sigma(x)]\}$ and $f^{*}[\sigma(x)]=\min \{f(x), f[\sigma(x)]\}$ for $x \in M_{+}$and functions defined everywhere on $M$. Then

$$
\int_{M \times M} \varphi\left[\frac{\left|f^{*}(x)-g^{*}(y)\right|}{\rho(x, y)}\right] k(x, y) d x d y \leq \int_{M \times M} \varphi\left[\frac{|f(x)-g(y)|}{\rho(x, y)}\right] k(x, y) d x d y
$$

The key to the proof of this two-point inequality lies with two simple observations. 
Lemma 4. For $\varphi$ convex with $\varphi(0)=0$ and nonnegative numerical sequences $\left\{a_{1}, a_{2}\right\}$ and $\left\{b_{1}, b_{2}\right\}$

$$
\varphi\left[\left|a_{1}-b_{1}\right|\right]+\varphi\left[\left|a_{2}-b_{2}\right|\right] \geq \varphi\left[\left|a_{1}^{*}-b_{1}^{*}\right|\right]+\varphi\left[\left|a_{2}^{*}-b_{2}^{*}\right|\right]
$$

Proof. This result is determined by applying the property of convex functions that slopes are increasing; that is, $\varphi(s+t)-\varphi(t)$ is increasing in $t$.

Lemma 5. For $\varphi$ convex, $\varphi(0)=0$ and $t \varphi^{\prime}(t)$ convex, then

$$
T(\lambda)=\varphi\left[\lambda\left|a_{1}-b_{1}\right|\right]+\varphi\left[\lambda\left|a_{2}-b_{2}\right|\right]-\varphi\left[\lambda\left|a_{1}^{*}-b_{1}^{*}\right|\right]-\varphi\left[\lambda\left|a_{2}^{*}-b_{2}^{*}\right|\right]
$$

is nondecreasing for $\lambda>0$.

Proof. Observe that $t \varphi^{\prime}(t)$ convex implies that $T^{\prime}(\lambda) \geq 0$ which determines that not only does $\varphi\left(\lambda\left|a_{1}-b_{1}\right|\right)+\varphi\left(\lambda\left|a_{2}-b_{2}\right|\right)$ not increase under rearrangement but that the variation does not decrease under scaling increase.

Proof.

$$
\begin{aligned}
\int_{M \times M} \varphi & {\left[\frac{|f(x)-g(y)|}{\rho(x, y)}\right] k(x, y) d x d y } \\
= & \int_{M_{+} \times M_{+}}\left[\left\{\varphi\left[\frac{f(x)-g(y)}{\rho(x, y)}\right]+\varphi\left[\frac{|f(\sigma(x))-g(\sigma(y))|}{\rho(x, y)}\right]\right\} k(x, y)\right. \\
& \left.+\left\{\varphi\left[\frac{|f(\sigma(x))-g(y)|}{\rho(x, \sigma(y))}\right]+\varphi\left[\frac{\mid f(x)-g(\sigma(y))}{\rho(x, \sigma(y))}\right]\right\} k(x, \sigma(y))\right] d x d y \\
= & \int_{M_{+} \times M_{+}}\left\{\varphi\left[\frac{|f(x)-g(y)|}{\rho(x, y)}\right]+\varphi\left[\frac{|f(\sigma(x))-g(\sigma(y))|}{\rho(x, y)}\right]\right\}[k(x, y)-(x, \sigma(y)] d x d y \\
& +\int_{M_{+} \times M_{+}}\left\{\varphi\left[\frac{|f(x)-g(y)|}{\rho(x, y)}\right]+\varphi\left[\frac{|f(\sigma(x))-g(\sigma(y))|}{\rho(x, y)}\right]-\varphi\left[\frac{|f(x)-g(y)|}{\rho(x, \sigma(y))}\right]\right. \\
& \left.-\varphi\left[\frac{|f(\sigma(x))-g(\sigma(y))|}{\rho(x, \sigma(y))}\right]\right\} k(x, \sigma(y)) d x d y \\
& +\int_{M_{+} \times M_{+}}\left[\left\{\varphi\left[\frac{|f(x)-g(y)|}{\rho(x, \sigma(y))}\right]+\varphi\left[\frac{|f(\sigma(x))-g(\sigma(y))|}{\rho(x, \sigma(y))}\right]+\varphi\left[\frac{|f(\sigma(x))-g(y)|}{\rho(x, \sigma(y))}\right]\right.\right. \\
& \left.+\varphi\left[\frac{|f(x)-g(\sigma(y))|}{\rho(x, \sigma(y))}\right]\right\} k(x, \sigma(y)) d x d y
\end{aligned}
$$

Since $\varphi$ is convex, the first integral decreases when $f$ and $g$ are replaced by $f^{*}$ and $g^{*}$ using Lemma 4, since $t \varphi^{\prime}(t)$ is convex, the second integral decreases when $f$ and $g$ are replaced by $f^{*}$ and $g^{*}$ using Lemma 5. Finally the third integral is invariant under symmetrization. Hence the proof of the Two-Point Symmetrization Lemma is complete by using simple point-wise estimates for the integrand.

Corollary (strict monotonicity). Assume that $k(x, y)>k[x, \sigma(y)]$ for $x, y \in M_{+}, \varphi$ is strictly convex, and there exist sets of positive measure $A$ and $B$ in $M_{+}$with $f(x)>f[\sigma(x)]$ for $x \in A$ and $g(x)<g[\sigma(x)]$ for $x \in B$. Then inequality (32) becomes a strict inequality.

To complete the proof of the Symmetrization Lemma for the classical manifolds $\mathbb{R}^{n}, S^{n}$ and $\mathbb{H}^{n}$ (hyperbolic space), the two curved spaces can be represented as sitting in $\mathbb{R}^{n+1}$ with a 
preferred point of reference, and a Euclidean half-space is specified so that reflection across the half-space has the required property for the metric - namely, reflection symmetry increases distance between points in the sense that

$$
\operatorname{dist}(x, y) \leq \operatorname{dist}(x, \sigma(y))
$$

for two points $x$ and $y$ in the specified half-space.

Symmetrization Lemma. Let $M$ be a geometric manifold that possesses: 1) a transitive group action under which the metric is invariant, and 2) reflection symmetry as defined above. Equimeasurable radial decreasing rearrangement is defined in terms of geodesic distance. Let $\varphi, K, \rho$ be nonnegative functions defined on $[0, \infty)$ with the following properties: (i) $\varphi(0)=0, \varphi$ convex and monotone increasing, $\varphi^{\prime \prime} \geq 0$, and $t \varphi^{\prime}(t)$ convex, (ii) $K$ monotone decreasing, and (iii) $\rho$ monotone increasing; and $d(x, y)$ is the distance between $x$ and $y$. Then for measurable functions $f$ and $g$

$$
\begin{aligned}
& \int_{M \times M} \varphi\left[\frac{|f(x)-f(y)|}{\rho[d(x, y)]}\right] K[d(x, y)] d x d y \\
& \quad \geq \int_{M \times M} \varphi\left[\frac{\left|f^{*}(x)-f^{*}(y)\right|}{\rho[d(x, y)]}\right] K[d(x, y)] d x d y
\end{aligned}
$$

If $K$ is strictly decreasing and $\varphi$ is strictly convex, then strict inequality holds unless $f(x)=$ $\lambda f^{*}(\tau x)$ and $g(x)=\lambda g^{*}(\tau x)$ with $|\lambda|=1$ and $\tau x$ a translate of $x$, or in the case of a finite measure one of the functions $f, g$ is constant almost everywhere. In the case $\rho$ is constant, the last condition in the hypothesis on $\varphi$ can be dropped.

Proof. Fix an origin in $M$ and choose a "hyperplane" that does not pass through this point. $M_{+}$ will denote the half-space containing the origin and $\sigma$ will be reflection through this hyperplane. By possibly changing values on a set of measure zero, $f$ and $g$ will be defined everywhere on $M$. Choose a sequence of two-point symmetrizations which when applied to $f, g$ give sequences $f_{n}, g_{n}$ that converge almost everywhere to the radial decreasing rearrangements $f^{*}, g^{*}$ on $M$. Each two-point symmetrization applied to a function gives a rearrangement of that function. The "two-point" lemma shows that

$$
\int_{M \times M} \varphi\left[\frac{\left|f_{n}(x)-g_{n}(y)\right|}{\rho[d(x, y)]}\right] K[d(x, y)] d x d y
$$

is a decreasing sequence of numbers as $n \rightarrow \infty$. Applying Fatou's lemma to this sequence of non-negative $L^{1}(M \times M)$ functions gives inequality (35). If $f$ and $g$ are not almost everywhere radial decreasing functions with respect to the same fixed point on $M$, then the first two-point symmetrization can be chosen to give a strict decrease in the inequality. This fact is equivalent to the existence of a half-space $M_{+}$such that the set

$$
\left\{(x, y) \in M_{+} \times M_{+}:[f(x)-f(\sigma(x))][g(y)-g(\sigma(y))]<0\right\}
$$

has positive measure.

\section{ACKNOWLEDGEMENTS}

I would like to thank Nestor Guillen for drawing my interest to kinetic problems in Landau collision dynamics. In addition, I would like to thank Guozhen Lu for arranging my visit to Nanjing University where some parts of this paper were developed, and for drawing my attention to his recent work with Han and Zhu ([23]). Paul Garrett's notes on "Classical homogeneous spaces" provided useful background. 


\section{REFERENCES}

[1] L. Ahlfors, Conformal invariants: topics in geometric function theory, McGraw-Hill, New York, 1973.

[2] R. Alexandre, Y. Morimoto, S. Ukai, C-J. Xu and T. Yang, The Boltzmann equation without angular cutoff in the whole space: I. Global existence for soft potential, Jour. Funct. Anal. 262 (2012), 915-1010.

[3] A. Baernstein and B.A. Taylor, Spherical rearrangements, subharmonic functions, and $*$-functions in $n$ space, Duke Math. J. 43 (1976), 245-268.

[4] W. Beckner, Inequalities in Fourier Analysis, Ann. Math. 102 (1975), 159-182.

[5] W. Beckner, Sobolev inequalities, the Poisson semigroup and analysis on the sphere $S^{n}$, Proc. Nat. Acad. Sci. 89 (1992), 4816-4819.

[6] W. Beckner, Geometric inequalities in Fourier analysis, in: Essays on Fourier Analysis in Honor of Elias M. Stein, pp. 36-68, Princeton University Press, Princeton, 1995.

[7] W. Beckner, Pitt's inequality and the uncertainty principle, Proc. Amer. Math. Soc. 123 (1995), $1897-1905$.

[8] W. Beckner, Sharp inequalities and geometric manifolds, J. Fourier Anal. Appl. 3 (1997), 303-323.

[9] W. Beckner, Weighted inequalities and Stein-Weiss potentials, Forum Math. 20 (2008), 587-606.

[10] W. Beckner, Pitt's inequality with sharp convolution estimates, Proc. Amer. Math. Soc. 136 (2008), 18711885.

[11] W. Beckner, Pitt's inequality and the fractional Laplacian: sharp error estimates, Forum Math 24 (2012), 177-209.

[12] W. Beckner, Multilinear embedding estimates for the fractional Laplacian, Math. Res. Lett. 19 (2012), 175189.

[13] W. Beckner, Analysis on Lie groups - embedding potentials, preprint.

[14] O.V. Besov, Investigation of a family of function spaces in connection with theorems of imbedding and extension, Trudy Mat. Inst. Stekov 60 (1961), 42-81; Amer. Math. Soc. Transl. 40 (1964), 85-126.

[15] J. Bourgain, H. Brezis and P. Mironescu, Another look at Sobolev spaces, in Optimal Control and Partial Differential Equations - Innovations and Applications, pp. 439-455, IOS Press, Amsterdam, 2000.

[16] J. Bourgain, H. Brezis and P. Mironescu, Limiting embedding theorems for $W^{s, p}$ when $s \uparrow 1$ and applications, J. Anal. Math. 87 (2002), 77-101.

[17] D.V. Chudnovsky, G.V. Chudnovsky and T. Morgan, Variance of signals and their finite Fourier transform, in Additive Number Theory, Springer-Verlag, New York, 2010, pp. 53-76.

[18] P. Constantin and V. Vicol, Nonlinear maximum principles for dissipative linear nonlocal operators and applications, arXiv: 1110.0179 .

[19] L. Desvillettes, Plasma kinetic models: the Fokker-Planck-Landau equation, in Modeling and computational methods for kinetic equations, Birkhäuser, Boston, 2004, pp. 171-193.

[20] R.L. Frank, E.H. Lieb and R. Seiringer, Hardy-Lieb-Thirring inequalities for fractional Schrödinger operators, J. Amer. Math. Soc. 21 (2008), 925-950.

[21] R.L. Frank and R. Seiringer, Non-linear ground state representations and sharp Hardy inequalities, J. Funct. Anal. 25 (2008), 3407-3430.

[22] P.T. Gressman and R.M. Strain, Global classical solutions of the Boltzmann equation without angular cut-off, J. Amer. Math. Soc. 24 (2011), 771-847.

[23] X. Han, G. Lu and J. Zhu, Hardy-Littlewood-Sobolev and Stein-Weiss inequalities on the Heisenberg group, Nonlinear Analysis 75 (2012), 4296-4314.

[24] G.H. Hardy and J.E. Littlewood, A maximal theorem with function-theoretic applications, Acta Math. 54 (1930), 81-116.

[25] G.H. Hardy, J.E. Littlewood and G. Pólya, Inequalities, Cambridge University Press, London, 1967.

[26] L.D. Landau, The transport equation in the case of Coulomb interactions, in Collected Papers of L.D. Landau, Gordon and Breach, New York, 1965, pp. 163-170.

[27] E.H. Lieb, Sharp constants in the Hardy-Littlewood-Sobolev and related inequalities, Ann. Math. 118 (1983), 349-374.

[28] E.M. Lifshitz and L.P. Pitaevskii, Physical kinetics, Pergamon Press, Oxford, 1981.

[29] P.-L. Lions, On Boltzmann and Landau equations, Phil. Trans. Royal Soc. Lond. A 346 (1994), $191-204$.

[30] V.G. Maz'ya, Sobolev spaces (2nd ed.), Springer-Verlag, 2011.

[31] V.G. Maz'ya and T. Shaposhnikova, On the Bourgain, Brezis and Mironescu theorem concerning limiting embeddings of fractional Sobolev spaces, J. Funct. Anal. 195 (2002), 230-238.

[32] E.M. Stein, Singular integrals and differentiability properties of functions, Princeton University Press, 1970.

[33] E.M. Stein, Some problems in harmonic analysis suggested by symmetric spaces and semi-simple groups, in Actes Congrès Intern. Math. (Nice, 1970), Vol. I, pp. 173-189, Gauthier-Villars, Paris, 1971. 
[34] C. Villani, On a new class of weak solutions to the spatially homogeneous Boltzmann and Landau equations, Arch. Rational Mech. Anal. 143 (1998), 273-307.

[35] A. Weil, L'integration dans les groupes topologiques et ses applications, Hermann, Paris, 1940.

Department of Mathematics, The University of Texas at Austin, 1 University Station C1200, Austin TX 78712-0257 USA

E-mail address: beckner@math.utexas.edu 